OPEN ACCESS

Edited by:

Guillermo Diaz-Araya,

University of Chile, Chile

Reviewed by:

Shankar Munusamy,

Drake University,

United States

Kazem Zibara,

Lebanese University, Lebanon

Ana Cristina Simões E. Silva, Federal University of Minas Gerais,

Brazil

${ }^{*}$ Correspondence:

Cristián A. Amador

cristian.amador@uautonoma.cl

Specialty section:

This article was submitted to

Translational Pharmacology,

a section of the journa

Frontiers in Pharmacology

Received: 01 July 2019

Accepted: 15 October 2019

Published: 14 November 2019

Citation:

Figueroa SM, Lozano M, Lobos C, Hennrikus MT, Gonzalez AA and Amador CA (2019) Upregulation of Cortical Renin and Downregulation of Medullary (Pro)Renin Receptor in

Unilateral Ureteral Obstruction.

Front. Pharmacol. 10:1314

doi: 10.3389/fphar.2019.01314

\section{Upregulation of Cortical Renin and Downregulation of Medullary (Pro) Renin Receptor in Unilateral Ureteral Obstruction}

\author{
Stefanny M. Figueroa ${ }^{1,2}$, Mauricio Lozano ${ }^{1}$, Carolina Lobos ${ }^{1}$, Matthew T. Hennrikus ${ }^{3}$, \\ Alexis A. Gonzalez ${ }^{2}$ and Cristián A. Amador ${ }^{1 *}$ \\ ${ }^{1}$ Laboratorio de Fisiopatología Renal, Instituto de Ciencias Biomédicas, Universidad Autónoma de Chile, Santiago, Chile, \\ ${ }^{2}$ Instituto de Química, Pontificia Universidad Católica de Valparaíso, Valparaíso, Chile, ${ }^{3}$ Department of Physiology, Tulane \\ University School of Medicine, New Orleans, LA, United States
}

Chronic kidney disease (CKD) is characterized by renal dysfunction, which is a common feature of other major diseases, such as hypertension and diabetes. Unilateral ureteral obstruction (UUO) has been used as a model of CKD in experimental animals and consists of total obstruction of one kidney ureter. The UUO decreases renal blood flow, which promotes the synthesis of renin in the juxtaglomerular apparatus, the first step in renin-angiotensin system (RAS) cascade. RAS induces inflammation and remodeling, along with reduced renal function. However, it remains unknown whether intrarenal RAS (iRAS) is activated in early stages of CKD. Our objective was to characterize different iRAS components in the renal cortex and in the medulla in an early phase of UUO. Male C57BL/6 mice (8-12 weeks old) were subjected to UUO in the left kidney, or to sham surgery, and were euthanized after 7 days ( $n=5 /$ group). Renal function, renal inflammatory/remodeling processes, and iRAS expression were evaluated. UUO increased plasma creatinine, right renal hypertrophy $(9.08 \pm 0.31, P<0.05 \mathrm{vs}$. Sham), and tubular dilatation in the left kidney cortex (42.42 $\pm 8.19 \mu \mathrm{m}, P<0.05 \mathrm{vs}$. Sham). This correlated with the increased mRNA of IL-1 $\beta(1.73 \pm 0.14, P<0.01$ vs. Sham, a pro-inflammatory cytokine) and TGF- $\beta 1$ (1.76 \pm $0.10, P<0.001$ vs. Sham, a pro-fibrotic marker). In the renal cortex of the left kidney, UUO increased the mRNA and protein levels of renin (in 35\% and 28\%, respectively, $\mathrm{P}<$ 0.05 vs. Sham). UUO decreased mRNA and protein levels for the (pro)renin receptor in the renal medulla $(0.67 \pm 0.036$ and $0.88 \pm 0.028$, respectively, $P<0.05$ vs. Sham). Our results suggest that modulation of iRAS components depends on renal localization and occurs in parallel with remodeling and pro-inflammatory/pro-fibrotic mechanisms.

Keywords: chronic kidney disease, intrarenal RAS, unilateral ureter obstruction, inflammation, pro-renin receptor

\section{INTRODUCTION}

Chronic kidney disease (CKD) is a worldwide health problem closely linked to other major diseases (Tonelli et al., 2015) that is difficult to diagnose and affects around 10\% of the population (De Nicola and Zoccali 2016). CKD increases cardiovascular mortality and is a risk multiplier for patients with hypertension and/or diabetes mellitus (Couser et al., 2011). 
CKD is defined by a sustained reduction in glomerular filtration rate (GFR) (Levey et al., 2011), which involves structural or functional anomalies of the kidneys evidenced on urine analysis (proteinuria) (Tonelli et al., 2011), biopsy, or imaging. Evidential structural abnormalities in CKD are characterized by glomerulosclerosis, tubular atrophy, and tubulo-interstitial fibrosis (Menon and Ross 2016), three conditions that drive nephrons to deterioration and to irreversible renal failure.

The unilateral ureteral obstruction (UUO) is a physiologically relevant in vivo model of renal inflammation that leads to fibrosis (Yang et al., 2010). UUO recapitulates the fundamental pathophysiologic mechanisms that typify all forms of human CKD in a relatively short time span (Eddy et al., 2012). It has been demonstrated that UUO increases interstitial capillary permeability (Yamaguchi et al., 2012) and the levels of IL-1-linked cytokines and transforming growth factor- $\beta 1$ (TGF- $\beta 1$ ), which correlate with the recruitment of inflammatory mononuclear cells (Eddy and Neilson 2006; Chi et al., 2017). Cao et al., 2015 described that during the first week, this pro-inflammatory phenotype is crucial for renal fibrosis consolidation observed after 4 weeks of UUO in mice (Cao et al., 2015). Thus, the UUO surgery in rodents can be used as an experimental model for studying the early (inflammatory, 7 days) (Ucero et al., 2014) and/or consolidated (fibrotic, 14 days) stage of CKD (MartínezKlimova et al., 2019).

Ureteral constriction reduces the GFR and induces a renin release (Eide et al., 1977), which represent the first step of renin-angiotensin system (RAS) activation (Kobori et al., 2007). This effect is considered to be critical because kidneys possess an intrinsic RAS (or intrarenal RAS, iRAS) that can also regulate the water and sodium balance and blood pressure (Velez 2009). In this context, it has been demonstrated that $24 \mathrm{~h}$ of UUO downregulates the mRNA levels of renal angiotensin-II (AngII) type 1 receptor $\left(\mathrm{AT}_{1} \mathrm{R}\right)$ (Pimentel et al.,1994) and increases the mRNA levels of renin from the juxtaglomerular apparatus (Pimentel et al., 1993). These changes, of which most were characterized 20 years ago, suggest that obstructive uropathy rapidly leads to hemodynamic alterations that may be implicated in the onset of CKD. Renin is also expressed in the collecting duct and is secreted by the principal cells in the renal medulla. Renin binds to the (pro)renin receptor (PRR, named also ATPase, $\mathrm{H}^{+}$transporting lysosomal accessory protein 2-Atp6ap2) (Nehme et al., 2019) at the neighboring intercalated cells (also in the medulla), thus activating pro-renin in a catalytic or non-catalytic form and increasing intratubular AngII formation while also triggering intracellular cascades related to fibrosis (Gonzalez et al., 2017).

We hypothesized that iRAS activation occurs during the early structural changes and the pro-inflammatory phase induced by UUO. This would be crucial for fibrosis consolidation and renal remodeling. Therefore, the main objective of our work was to characterize the iRAS components such as renin and PRR in the renal cortex and in the medulla at 7 days of UUO in mice.

\section{MATERIALS AND METHODS}

\section{Animals and Experimental Protocol}

For UUO, 9- to 12-week-old male C57BL/6 mice $(n=5)$ were anesthetized with a solution of ketamine/xylazine (80:8 $\mathrm{mg} \mathrm{kg}^{-1}$ ) and the left ureter was exposed by a dorsal incision. Then the ureter was obstructed by two-point ligation with silk sutures. Sham-operated mice $(n=5)$ underwent the same procedure, except for the obstruction of the left ureter (Ucero et al., 2014). All the experimental procedures with animals were performed according to the Committee on Animal Research and Ethics (Guidelines for Ethical Conduct in the Care and Use of Nonhuman Animals in Research, 2012) and were under the surveillance of the Ethics Committee of the Universidad Autonoma de Chile. Animals were placed in conditions of light-dark cycle $(12 \mathrm{~h})$, temperature of $21^{\circ} \mathrm{C}$, humidity of $50 \%$, adequate ventilation, noise-free, food and water ad libitum, and under continuous veterinary monitoring.

For histological and biochemical analyses, the animals were sacrificed 7 days after surgeries and the kidneys were quickly removed through a dorsal incision. Plasma was collected and stored at $-20^{\circ} \mathrm{C}$. Plasma creatinine and blood urine nitrogen (BUN) were analyzed using automated chemistry Jaffe assay and UV urease kit, respectively (Valtek Diagnostics, Santiago, Chile).

\section{Histological Analysis}

Sagittal sections of obstructed kidneys were fixed in Bouin (Sigma Aldrich, USA), embedded in paraffin, and 5- $\mu \mathrm{m}$ sections were prepared using a Leica RM2235 microtome (Leica Microsystems, Shanghai). The sections were washed and stained with hematoxylin-eosin (Sigma Aldrich, USA) for the assessment of tubular diameter. Images for cortices and medullas were captured with a Nikon digital sight DS-U3 digital camera, attached to a Nikon Upright Microscope ECLIPSE Ci-L (Nikon Instruments Inc., USA), and analyzed using Image $1.43 \mathrm{u}$ software (NIH, USA).

\section{Total RNA Isolation and qrt-PCR}

Total RNA from whole kidney, cortex, and medulla was extracted with the TRIzol reagent, according to the manufacturer's instructions (ThermoFisher Scientific, USA). For whole kidney and renal cortex, cDNA was synthesized from $2 \mu \mathrm{g}$ of RNA using M-MLV reverse transcriptase kit (Promega, USA), while in the medulla, cDNA was synthesized from $1 \mu \mathrm{g}$ of RNA using Superscript II reverse transcriptase kit (ThermoFisher Scientific, USA). Real-time PCR reactions were performed in a LightCycler96 (Roche, Switzerland), and transcript levels were detected by SYBR Green method using FastStart Essential DNA Master mix (Roche, Switzerland). The following primers were used: 18S: (F) 5'-GCC GCTAGAGGTGAAATTCTTGGA-3', (R) 5'-ATCGCCAGTGGCATCGTTTAT-3'; angiotensin-converting enzyme (ACE)1: (F) 5' -ATGAAGCCAAGGCTGACAGGTT-3', (R) 5'-GTGATTGGAT ACCTCCGTTGCTT-3'; ACE2: (F) 5'-GGGGTGAAATTCCCAAAGAGCAGT-3'，(R) 5'-TCTGC CCAGCTTCAGTGGAATTTG-3'; AGT: (F) 5'-ACTGCTCC 
AGGCTTTCGTCTAA-3', (R) 5'-TC CACCCTGTCACAGCCT TTAT-3'; AT1Ra: (F) 5'-ACACTGCCATGCCCATAACCA3', (R) 5'-T AGACAGGCTTGAGTGCGACTT-3'; IL-1 $\beta$ : (F) 5'-ACTGGTACATCAGCACCTCACAAG-3', (R) 5'-GCT GTCTGCTCATTCACGAAAAGG-3'; PRR (Atp6ap2 gene): (F) 5'-GCGGGTGCTTTA GGGAATGAAT-3', (R) 5'-TGG CCAAGACAGGTCTTCCTTT-3'; REN1: (F) 5'-AGCTCCCT GA AGTTGATCATGC-3', (R) 5'-CTCCTGTTGGGATACTGT AGCA-3'; TGF- $\beta 1$ : (F) 5'-TACGCCT GAGTGGCTGTCTTTT3', (R) 5'-TTGGGGCTGATCCCGTTGATTT-3'. All PCR products were subjected to a melting curve program to confirm amplification specificity. Results were analyzed according to the standard curve method and mRNA level was calculated with respect to the relative amount of $18 \mathrm{~S}$ for each sample.

\section{Protein Analysis by Western Blot}

For protein extraction, a lysis buffer containing $50 \mathrm{mM}$ Tris$\mathrm{HCl}$ (pH 7.5), $0.1 \mathrm{mM} \mathrm{NaCl}, 0.5 \%$ Triton X-100, 0.5 M EDTA, and a mixture of protease/phosphatase inhibitors (Roche, Switzerland) were used. We used a D-160 Scilogex polytron (ABC Scientific, USA) and sonication at $130 \mathrm{~W}, 20 \mathrm{KHz}$ and $60 \%$ amplification (Ultrasonic Processor, USA) to homogenize the tissues. After centrifugation at 7,500 $\times g$ for $5 \mathrm{~min}$ at $4^{\circ} \mathrm{C}$, the supernatant was collected and total protein concentration in the sample was measured using a BCA protein assay kit (ThermoFisher Scientific, USA). Samples containing equal amounts of protein ( $40 \mu \mathrm{g}$ for cortex and $10 \mu \mathrm{g}$ for medulla/ lane) were separated based on their molecular weight by $7.5 \%$ polyacrylamide gels for electrophoresis (SDS-PAGE) and transferred onto nitrocellulose membranes at a constant current of $2.5 \mathrm{~A}, 25 \mathrm{~V}$ for $7 \mathrm{~min}$ (BioRad, USA). After blocking with $5 \%$ non-fat milk in Tris-buffered saline with $0.1 \%$ Tween20 (TBST) for $1 \mathrm{~h}$ at room temperature (RT), the membranes were stain with ponceau red and later incubated with primary antibodies against renin and ACE (both used at 1:200; Santa Cruz Biotechnology, USA), angiotensinogen (AGT) (1:200, Immuno-Biological Laboratory, Japan), PRR (1:250, Sigma Aldrich, USA), and $\alpha$-tubulin (1:40,000, Sigma Aldrich, USA) overnight at $4^{\circ} \mathrm{C}$. Subsequently, the membranes were washed with TBST and incubated with the corresponding secondary antibodies (including goat anti-mouse IgG and mouse antirabbit $\operatorname{IgG}, 1: 3,000$ ) for $1 \mathrm{~h}$ at $\mathrm{RT}$, and finally visualized by enhanced chemiluminescence reagents (ThermoFisher Scientific, USA). The relative expression level of proteins was analyzed using ImageJ software.

\section{Statistics}

Results from in vivo studies were expressed as the mean \pm SEM. Data were analyzed by one-way analysis of variance (ANOVA) test followed by Bonferroni posttest (more than two groups), by Student's $t$ test, or Mann-Whitney nonparametric test (two groups), as appropriate. All analyses were performed using GraphPad Prism (GraphPad Software, USA). Values of $P<0.05$ were considered as statistically significant.

\section{RESULTS}

\section{UUO Induces an Increase in Plasma Creatinine and Hypertrophy in Contralateral Kidney}

After 7 days of total left ureteral obstruction, mice presented with right kidney hypertrophy, as shown by the increase in right kidney weight/tibia length ratio $(9.76 \pm 0.42 \mathrm{~g}, P<0.05$; Table 1). No difference was detected in left kidney weight.

As an indirect way to determine GFR reduction in our model, we observed that UUO induced an increase in plasma creatinine in relation to the Sham group $(0.57 \pm 0.12 \mathrm{mg} / \mathrm{dl}, P<0.05)$, but did not increase the BUN.

\section{UUO Produces a Pro-Inflammatory and Pro-Fibrotic Status in the Obstructed Kidney}

Since our aim was to study the UUO during the proinflammatory phase leading to the pro-fibrotic phenotype, we determined the levels of interleukin-1 $\beta$ (IL-1 $\beta$ ) and TGF- $\beta 1$, as a major pro-inflammatory cytokine involved in tubulointerstitial injury (Tesch et al., 1997) and as the main fibrosis-promoting factor (Ding et al., 2014), respectively. The analysis by quantitative RT-PCR demonstrated that IL-1 $\beta$ (1.7-fold change, FC, vs. Sham, $P<0.01)$ and TGF- $\beta 1$ (1.9 FC vs. Sham, $P<0.001)$ were increased in the left (obstructed) kidney after 7 days of UUO (Figures 1A, B, respectively). These effects were not observed at level of the right kidney and were in concordance with previous results reported (Pimentel et al., 1995; Wang et al., 2015).

\section{UUO Induces Tubular Remodeling in Cortex and Medulla of Obstructed Kidney}

In order to explore the tubular remodeling induced by UUO in the renal cortex and in the medulla, we analyzed by light microscopy transversal sections of the left kidney stained by hematoxylin-eosin (Figure 2). We observed that UUO induced an increase of tubule interstitial space (Figure 2, black arrows) and an enlargement of diameter in cortical tubules (Figure 2), which has been related with the increase of the tubular lumen

TABLE 1 | UUO induces a contralateral renal hypertrophy and a rise of plasma creatinine.

\begin{tabular}{lcc}
\hline Group & Sham & UUO \\
\hline Total weight $(\mathrm{g})$ & $23.77 \pm 0.45$ & $22.28 \pm 0.61$ \\
Left kidney weight/tibia length $(\mathrm{mg} / \mathrm{mm})$ & $8.09 \pm 0.35$ & $8.41 \pm 0.38$ \\
Right kidney weight/tibia length $(\mathrm{mg} / \mathrm{mm})$ & $8.13 \pm 0.26$ & $\mathbf{9 . 7 6} \pm \mathbf{0 . 4 2}$ \\
Blood urea nitrogen $(\mathrm{mg} / \mathrm{dl})$ & $58.32 \pm 1.87$ & $59.19 \pm 7.49$ \\
Creatinine $(\mathrm{mg} / \mathrm{dl})$ & $0.368 \pm 0.021$ & $\mathbf{0 . 5 7 4} \pm \mathbf{0 . 1 1 7}$
\end{tabular}

Physiological parameters at 7 days of UUO BUN blood urea nitrogen, UUO unilateral ureteral obstruction Mann-Whitney statistic test. ${ }^{*} P<0.05$ vs. Sham group $(n=5)$. 


\section{A}

IL-1 $\beta$

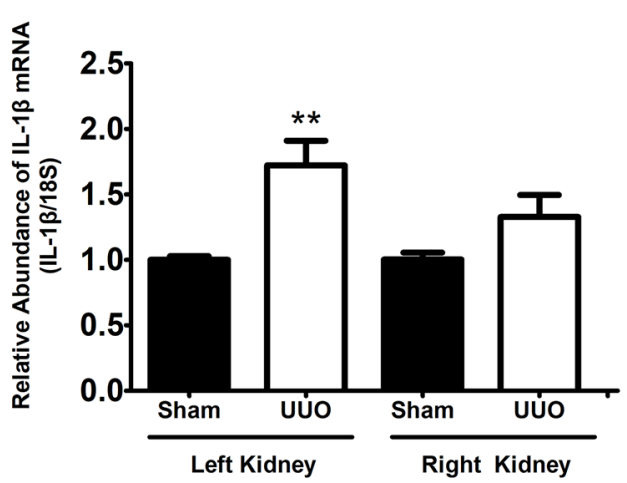

B

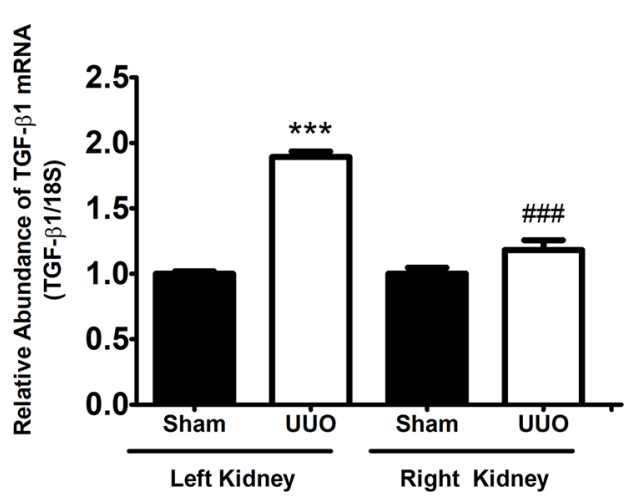

FIGURE 1 | Unilateral ureteral obstruction (UUO) induces a pro-inflammatory and pro-fibrotic phenotype at 7 days. (A) IL-1 $\beta$ and (B) TGF- $\beta 1$ mRNA levels in the left and right kidneys. 18S mRNA was used as the housekeeping gene. One-way ANOVA test followed by Bonferroni test was performed. ${ }^{\star *} P 0.01$ and ${ }^{\star \star *} P 0.001$ vs. Sham group. \#\#P 0.001 vs. UUO left kidney group $(n=5)$.

A

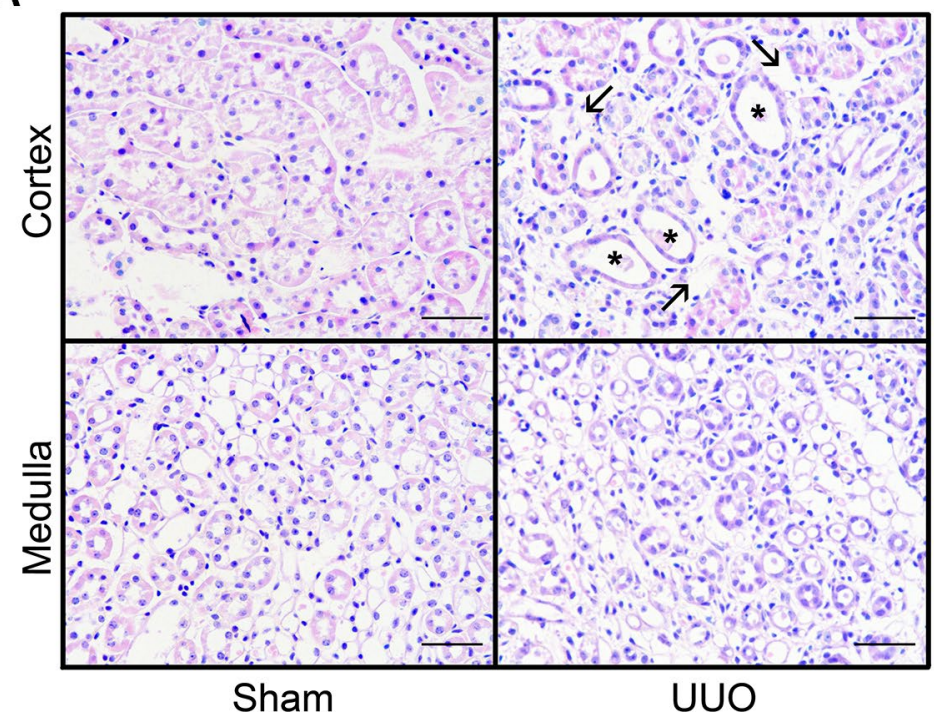

B

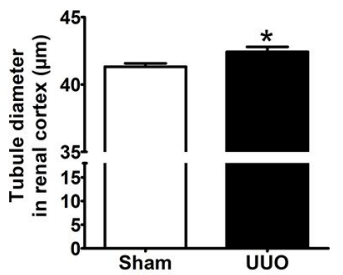

C

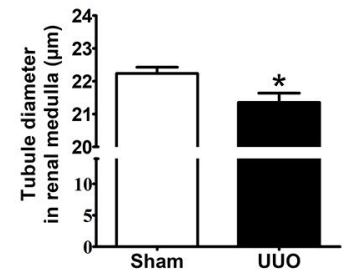

FIGURE 2 | Unilateral ureteral obstruction (UUO) induces tubular remodeling in the renal cortex and medulla after 7 days. (A) Haematoxylin-eosin staining in the renal cortex and medulla (scale bar, $100 \mu \mathrm{m}$ ). Black arrows show the increase of tubule interstitial space, while black asterisks show tubular dilation. Quantification of tubule diameter in the (B) cortex and $\mathbf{( C )}$ medulla of Sham and UUO groups. Mann-Whitney statistic test. ${ }^{\star} P 0.05$ vs. Sham group $(n=5)$.

area in obstructive uropathy $(P<0.05)$ (Cavaglieri et al., 2015). On the other hand, we detected a diminution in tubular diameter of kidney medulla with ureteral obstruction $(P<0.05)$ (Figure 2), suggesting a differential remodeling of kidney at 7 days of UUO.

\section{UUO Induces Differential Changes of iRAS Components in Left Renal Cortex and Medulla at mRNa Level, but not at Protein Level}

As the renal remodeling induced by 7 days of UUO was not equal in the renal cortex and in the medulla, we studied the levels of iRAS components separately. In the renal cortex of the left kidney, UUO promoted an induction of renin (1.4 FC vs. Sham, $P<0.001)$ and a reduction of ACE1 mRNA (0.3 FC vs. Sham, $P<0.01$ ) (Figure 3A), suggesting a differential response of iRAS. Only the renin increase was confirmed at protein level (Figure 3B). No differences were detected for the mRNA levels of AGT, ACE2, PRR, or AT1R in the renal cortex (Figure 3A), and neither for ACE1 at protein level (Figure 3C).

On the other hand, UUO diminished significantly the mRNA level for AGT, for ACE2 (in both cases 0.5 FC vs. Sham, $P<0.05)$, and for PRR $(0.3 \mathrm{FC}$ vs. Sham, $P<0.01)$ in renal medulla of the left kidney (Figure 4A). In the case of protein 

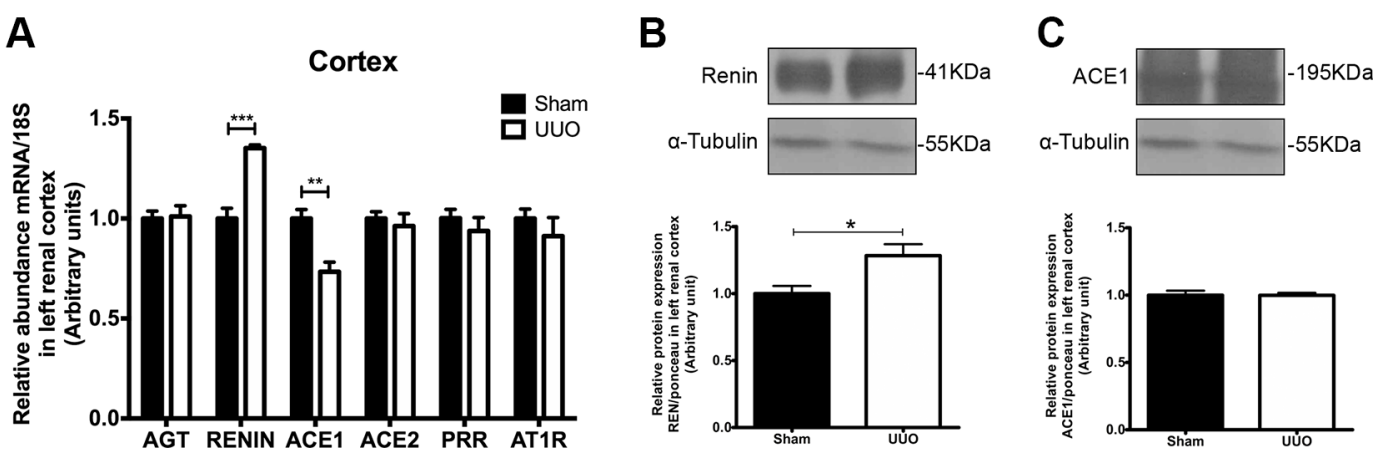

FIGURE 3 | Unilateral ureteral obstruction (UUO) induces upregulation of renin in left renal cortex. (A) mRNA levels for: angiotensinogen (AGT), renin, angiotensinconverting enzyme (ACE)1/2, (pro)renin receptor (PRR), and angiotensin-II receptor type 1 (AT1R) in the renal cortex. 18S mRNA was used as the housekeeping gene in qRT-PCR studies. Western blot and immunodetection of (B) renin and (C) ACE1 in the renal cortex. $\alpha$-tubulin was used as internal loading control. Student's $t$ test was performed. ${ }^{* \star} P<0.01$ or ${ }^{\star * \star} P<0.001$ vs. Sham group $(n=5)$.
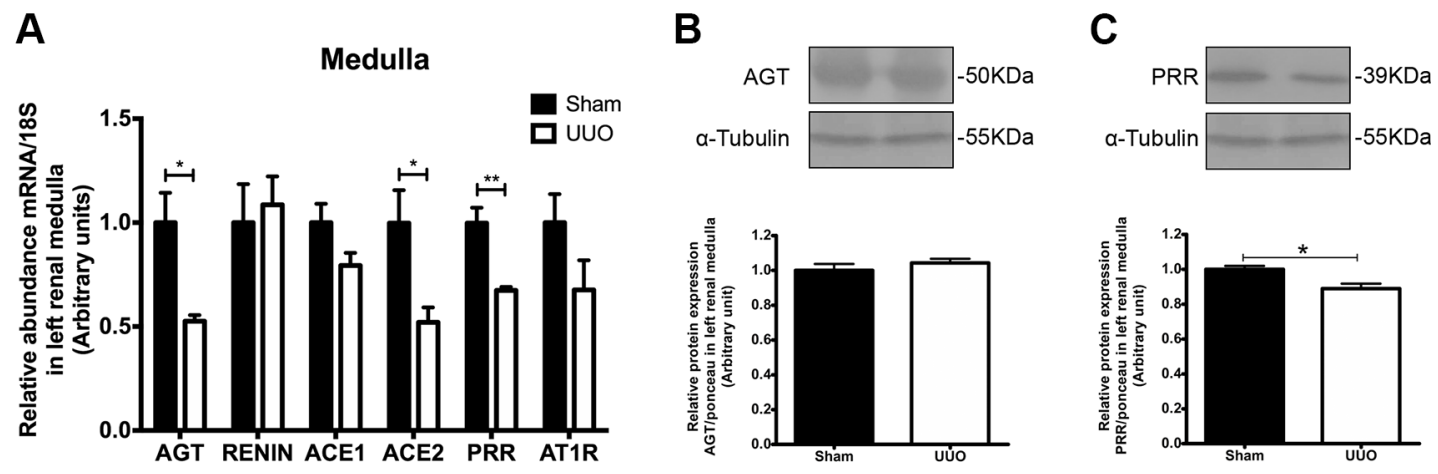

FIGURE 4 | UUO induces downregulation of (pro)renin receptor (PRR) in left renal medulla. (A) mRNA levels for: AGT, renin, ACE1/2, PRR, and AT1R in renal medulla. 18S mRNA was used as the housekeeping gene in qRT-PCR studies. Western blot and immunodetection of (B) AGT and (C) PRR in renal medulla. $\alpha$-tubulin was used as internal loading control. Student's $t$ test was performed. ${ }^{\star} P<0.05$ or ${ }^{\star \star} P<0.01$ vs. Sham group $(n=5)$.

expression, we corroborated the diminution only for PRR in renal medulla after UUO (Figures 4B, C). No differences were detected in medullary renin or ACE1 $(P=0.09)$. In the case of AT1R, we observed a modest reduction of mRNA (Figure 4A), which has been reported previously by Pimentel et al. at $24 \mathrm{~h}$ of UUO and by non-quantitative techniques (Pimentel et al.,1994).

\section{DISCUSSION}

Different mediators belonging to the RAS have been described in renal tissue for more than 30 years where their activation may promote or accelerate CKD. For instance, it has been demonstrated that in diabetes mellitus and hypertension, the two major risk factors of CKD, the urinary AGT (Hollenberg et al., 2003) and the intrarenal AngII (Navar et al., 2011), are induced. Here, by using an experimental CKD model that promotes renal inflammation driving to fibrosis, we have characterized different elements of iRAS in the cortex and in renal medulla.
Our main findings suggested that during the proinflammatory phase of obstructive uropathy, where the tubular remodeling is also observed, the iRAS is activated in the cortex as reflected by the increase in renin in dilated proximal tubules (Figure 5). This observation is in accordance with studies from Eide and collaborators, which demonstrated that ureteral constriction in dogs induces rapid renin release (Eide et al., 1977). In this study, renin upregulation was observed at mRNA and protein levels specifically in the cortex of obstructed kidneys after 7 days (Figure 3). An additional way for confirming an "iRAS activation" is the measurement of AngII; it has been demonstrated that $15 \mathrm{~h}$ of UUO in dogs increases the plasma AngII (Frøkiaer et al., 1992). However, this has not been demonstrated directly in kidney tubules of mice with ureteral obstruction due to the amount of tubular fluid necessary for AngII analysis. Alternatively, the increase of urinary AngII has been used as a marker of iRAS activation during CKD development in rats (Fang et al., 2018). In general, this iRAS activation results in hemodynamic changes with GFR reduction, observed as a plasma creatinine increase in UUO mice and in increases in the intraluminal pressure leading to 
Normal Tubule

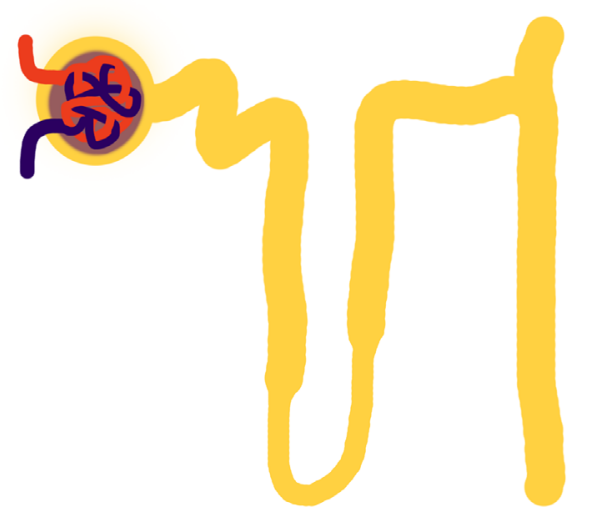

Obstructed Tubule

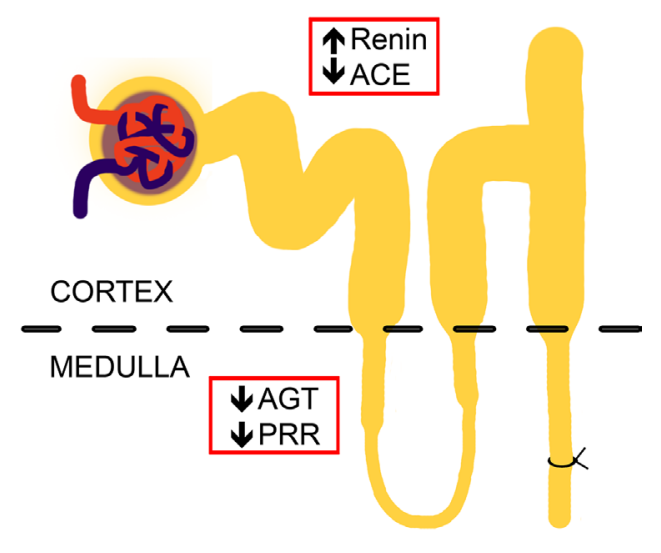

FIGURE 5 | Proposal of changes in intrarenal renin-angiotensin system (iRAS) components during the inflammatory stage of UUO. Obstructive uropathy at 7 days (recognized as "the pro-inflammatory phase") induces tubular remodeling and iRAS activation in the cortex, as reflected by the renin increase in dilated tubules. This activation may be counteracted by the iRAS components in the medulla, particularly by PRR.

tubular dilation observed in the proximal tubule (Table $\mathbf{1}$ and Figure 2, respectively).

Since renin and pro-renin are synthetized by the collecting ducts cells in kidneys, it has been hypothesized that AngII production has a crucial role in renal compartments and a local biological activity (Nehme et al., 2019). The experimental evidence suggests that pro-renin and renin generation is critical for intratubular AngII formation and for interaction with PRR, promoting tubular damage and further intratubular AngII formation (Gonzalez et al., 2017). In this context, it is proposed that PRR activation contributes directly to the generation of some pro-inflammatory mediators (for instance, prostaglandin E2), as an additional mechanism for renal damage and remodeling (Carlsen et al., 2010; Gonzalez and Prieto 2015). Since renin is increased during the pro-inflammatory phase of UUO at the cortical level, we propose that the downregulation of medullary PRR, corroborated at mRNA and protein levels (Figure 4A), may act as a negative feedback of the axis. This represents an integrative iRAS modulation between the cortex and medulla (Figure 5).

Cumulative evidence supports the beneficial effects of direct renin antagonism in UUO models. It has been demonstrated that aliskiren prevents the renal aquaporin-2 downregulation (Wang et al., 2015) and prevents the interstitial fibrosis and collagen accumulation at renal level induced by 7 days of UUO (Chung et al., 2017). In addition, it has been reported that the anti-inflammatory and anti-fibrotic effects observed by aliskiren can be achieved alone or in combination with calcium channel blockers and valsartan (Wu et al., 2010). On the other hand, Fang and collaborators showed that the administration of PRO20, a decoy inhibitor of PRR, prevents renal increase of TGF- $\beta 1$, oxidative stress, and interstitial fibrosis in rats with albumin overload (Fang et al., 2018). They demonstrated that PRR antagonism dramatically reduces the proteinuria, which has been recognized as an independent risk factor of CKD progression. This has not been demonstrated with other renin receptors, such as the renin-binding protein (RnBP) nor the mannose 6-phosphate/insulin-like grow factor II (M6P/IGFII) receptor (Batenburg and Danser 2012). Recently, Nehme et al. have highlighted that the regulation of PRR and other mediators of non-classical systemic RAS, such as ACE2 (called "extended RAS", and also evaluated by us in Figures 3 and 4) (Nehme et al., 2015; Nehme et al., 2016), can be used for the development of more effective and selective pharmaceuticals therapies.

In summary, we demonstrate that the pro-inflammatory phase of UUO involves tubular damage, with cortical upregulation of renin (juxtaglomerular) and medullary PRR downregulation. This scenario suggests a differential iRAS modulation as part of the mechanisms involved in the early stages of CKD.

\section{DATA AVAILABILITY STATEMENT}

The raw data supporting the conclusions of this manuscript will be made available by the authors, without undue reservation, to any qualified researcher.

\section{ETHICS STATEMENT}

The animal study was reviewed and approved by Ethic Commitee of the Universidad Autónoma de Chile.

\section{AUTHOR CONTRIBUTIONS}

CA and AG designed the experiments. SF conducted most of the experiments and collected and analyzed the data. ML and CL conducted specific experiments and collected 
and analyzed their respective data. CA and SF wrote the manuscript, and AG contributed to its revision. MH wrote and revised the manuscript.

\section{FUNDING}

This work was supported by FONDECYT Iniciación \#11150542 and DIUA157-2019 project from Vicerrectoría de Investigación y Postgrado (VRIP) (CA). In addition, SF received support

\section{REFERENCES}

Batenburg, W. W., and Danser, A. H. (2012). (Pro)Renin and its receptors: pathophysiological implications. Clin. Sci. 123 (3), 121-133. doi: 10.1042/ CS20120042

Cao, Q., Harris, D., and Wang, Y. (2015). Macrophages in kidney injury, inflammation, and fibrosis. Physiol. (Bethesda Md.) 30 (3), 183-194. doi: 10.1152/physiol.00046.2014

Carlsen, I., Donohue, K. E., Jensen, A. M., Selzer, A. L., Chen, J., Dix, P., et al. (2010). Increased cyclooxygenase-2 expression and prostaglandin e2 production in pressurized renal medullary interstitial cells. Am. J. Physiol. Regulatory Integr. Comp. Physiol. 299 (3), R823-R831. doi: 10.1152/ajpregu.00544.2009

Cavaglieri, R. C., Day, R. T., Feliers, D., and Abboud, H. E. (2015). Metformin prevents renal interstitial fibrosis in mice with unilateral ureteral obstruction. Mol. Cell. Endocrinol. 412, 116-122. doi: 10.1016/j.mce.2015.06.006

Chi, H. H., Hua, K. F., Lin, Y. C., Chu, C. L., Hsieh, C. Y., Hsu, Y. J., et al. (2017). IL-36 Signaling facilitates activation of the NLRP3 inflammasome and IL-23/ IL-17 axis in renal inflammation and fibrosis. J. Am. Soc. Nephrology: JASN 28 (7), 2022-2037. doi: 10.1681/ASN.2016080840

Chung, S., Kim, S., Kim, M., Koh, E. S., Shin, S. J., Park, C. W., et al. (2017). Treatment combining aliskiren with paricalcitol is effective against progressive renal tubulointerstitial fibrosis via dual blockade of intrarenal renin. PloS One 12 (7), e0181757. doi: 10.1371/journal.pone.0181757

Couser, W. G., Remuzzi, G., Mendis, S., and Tonelli, M. (2011). The contribution of chronic kidney disease to the global burden of major noncommunicable diseases. Kidney Int. 80 (12), 1258-1270. doi: 10.1038/ki.2011.368

De Nicola, L., and Zoccali, C. (2016). Chronic kidney disease prevalence in the general population: heterogeneity and concerns. Nephrology Dialysis Transplantation: Official Publ. Eur. Dialysis Transplant Assoc. Eur. Renal Assoc. 31 (3), 331-335. doi: 10.1093/ndt/gfv427

Ding, Y., Kim, S. I., Lee, S. Y., Koo, J. K., Wang, Z., and Choi, M. E. (2014). Autophagy regulates tgf- $\beta$ expression and suppresses kidney fibrosis induced by unilateral ureteral obstruction. J. Am. Soc. Nephrology: JASN 25 (12), 28352846. doi: 10.1681/ASN.2013101068

Eddy, A., and Neilson, E. (2006). Chronic kidney disease progression. J. Am. Soc. Nephrology: JASN 17 (11), 2964-2966. doi: 10.1681/ASN.2006070704

Eddy, A., López-Guisa, J., Okamura, D., and Yamaguchi, I. (2012). Investigating mechanisms of chronic kidney disease in mouse models. Pediatr. Nephrology (Berlin Germany) 27 (8), 1233-1247. doi: 10.1007/s00467-011-1938-2

Eide, I., Loyning, E., Langård, O., and Kiil, F. (1977). Mechanism of renin release during acute ureteral constriction in dogs. Circulation Res. 40 (3), 293-299. doi: 10.1161/01.res.40.3.293

Fang, H., Deng, M., Zhang, L., Lu, A., Su, J., Xu, C., et al. (2018). Role of (pro)renin receptor in albumin overload-induced nephropathy in rats. Am. J. Physiol. Renal Physiol. 315 (6), F1759-F1768. doi: 10.1152/ ajprenal.00071.2018

Frøkiaer, J., Knudsen, L., Nielsen, A. S., Pedersen, E. B., and Djurhuus, J. C. (1992). Enhanced intrarenal angiotensin II generation in response to obstruction of the pig ureter.Am. J. Physiol. 263 (3 Pt2), F527-F533. doi: 10.1152/ajprenal.1992.263.3.F527

Gonzalez, A., and Prieto, M. (2015). Renin and the (Pro)renin receptor in the renal collecting duct. Clin. Exp. Pharmacol. Physiol. 42 (1), 14-21. doi: 10.1111/1440-1681.12319

Gonzalez, A., Zamora, L., Reyes-Martinez, C., Salinas-Parra, N., Roldan, N., Cuevas, C., et al. (2017). (Pro)Renin receptor activation increases profibrotic markers and fibroblast-like phenotype through MAPK-Dependent ROS from the scholarships for undergraduate (VRIEA-Pontificia Universidad Católica de Valparaíso, \#039.407/2017) and $\mathrm{PhD}$ students (VRIP-UA).

\section{ACKNOWLEDGMENTS}

We thank Mrs. Eliana Pino and Mrs. Pamela Torres for their technical and administrative assistance, respectively.

formation in mouse renal collecting duct cells. Clin. Exp. Pharmacol. Physiol. 44 (11), 1134-1144. doi: 10.1111/1440-1681.12813

Hollenberg, N. K., Price, D. A., Fisher, N. D., Lansang, M. C., Perkins, B., Gordon, M. S., et al. (2003). Glomerular hemodynamics and the renin-angiotensin system in patients with type 1 diabetes mellitus. Kidney Int. 63 (1), 172-178. doi: 10.1046/j.1523-1755.2003.00701.x

Kobori, H., Nangaku, M., Navar, L. G., and Nishiyama, A. (2007). The intrarenal renin-angiotensin system: from physiology to the pathobiology of hypertension and kidney disease. Pharmacological Rev. 59 (3), 251-287. doi: 10.1124/pr.59.3.3

Levey, A. S., de Jong, P. E., Coresh, J., El Nahas, M., Astor, B. C., Matsushita, K., et al. (2011). The definition, classification, and prognosis of chronic kidney disease: A KDIGO controversies conference Report. Kidney Int. 80 (1), 17-28. doi: $10.1038 / \mathrm{ki} .2010 .483$

Martínez-Klimova, E., Aparicio-Trejo, O. E., Tapia, E., and Pedraza-Chaverri, J. (2019). Unilateral ureteral obstruction as a model to investigate fibrosisattenuating treatments. Biomolecules 9 (4), 1-28. doi: 10.3390/biom9040141

Menon, M. C., and Ross, M. J. (2016). Epithelial-to-mesenchymal transition of tubular epithelial cells in renal fibrosis: a new twist on an old tale. Kidney Int. 89 (2), 263-266. doi: 10.1016/j.kint.2015.12.025

Navar, L. G., Kobori, H., Prieto, M. C., and Gonzalez-Villalobos, R. A. (2011). Intratubular renin-angiotensin system in hypertension. Hypertension 57 (3), 355-362. doi: 10.1161/HYPERTENSIONAHA.110.163519

Nehme, A., Cerutti, C., and Zibara, K. (2016). Transcriptomic analysis reveals novel transcription factors associated with renin-angiotensin-aldosterone system in human atheroma. Hypertension 68 (6), 1375-1384. doi: 10.1161/ HYPERTENSIONAHA.116.08070

Nehme, A., Cerutti, C., Dhaouadi, N., Gustin, M. P., Courand, P. Y., Zibara, K., et al. (2015). Atlas of tissue renin-angiotensin-aldosterone system in human: a transcriptomic meta-analysis. Sci. Rep. 5, 10035. doi: 10.1038/ srep 10035

Nehme, A., Zouein, F. A., Zayeri, Z. D., and Zibara, K. (2019). An update on the tissue renin angiotensin system and its role in physiology and pathology. J. Cardiovasc. Dev. Dis. 6 (2), 1-17. doi: 10.3390/jcdd6020014

Nehme, A., Marcelo, P., Nasser, R., Kobeissy, F., Bricca, G., and Zibara, K. (2016b). The kinetics of angiotensin-I metabolism in human carotid atheroma: an emerging role for angiotensin (1-7). Vascular Pharmacol. 85, 50-56. doi: 10.1016/j.vph.2016.08.001

Pimentel, J. L., Sundell, C. L., Wang, S., Kopp, J. B., Montero, A., and MartínezMaldonado, M. (1995). Role of angiotensin II in the expression and regulation of transforming growth factor-beta in obstructive nephropathy. Kidney Int. 48 (4), 1233-1246. doi: 10.1038/ki.1995.407

Pimentel, J. L., Martinez-Maldonado, M., Wilcox, J. N., Wang, S., and Luo, C. (1993). Regulation of renin-angiotensin system in unilateral ureteral obstruction. Kidney Int. 44 (2), 390-400. doi: 10.1038/ki.1993.257

Pimentel, J. L., Wang, S., and Martinez-Maldonado, M. (1994). Regulation of the renal angiotensin II receptor gene in acute unilateral ureteral obstruction. Kidney Int. 45 (6), 1614-1621. doi: 10.1038/ki.1994.212

Tesch, G. H., Yang, N., Yu, H., Lan, H. Y., Foti, R., Chadban, S. J., et al. (1997). Intrinsic renal cells are the major source of interleukin-1 beta synthesis in normal and diseased rat kidney. Nephrology Dialysis Transplantation: Official Publ. Eur. Dialysis Transplant Assoc. Eur. Renal Assoc. 12 (6), 1109-1115. doi: 10.1093/ndt/12.6.1109

Tonelli, M., Wiebe, N., Guthrie, B., James, M. T., Quan, H., Fortin, M., et al. (2015). Comorbidity as a driver of adverse outcomes in people with chronic kidney disease. Kidney Int. 88 (4), 859-866. doi: 10.1038/ki.2015.228 
Tonelli, M., Muntner, P., Lloyd, A., Manns, B. J., James, M. T., Klarenbach, S., et al. (2011). Using proteinuria and estimated glomerular filtration rate to classify risk in patients with chronic kidney disease: a cohort study. Ann. Internal Med. 154 (1), 12-21. doi: 10.7326/0003-4819-154-1-201101040-00003

Ucero, A. C., Benito-Martin, A., Izquierdo, M. C., Sanchez-Niño, M. D., Sanz, A. B., Ramos, A. M., et al. (2014). Unilateral ureteral obstruction: beyond obstruction. Int. Urology Nephrology 46 (4), 765-776. doi: 10.1007/s11255-013-0520-1

Velez, J. C. (2009). The importance of the intrarenal renin-angiotensin system. Nat. Clin. Pract. Nephrology 5 (2), 89-100. doi: 10.1038/ncpneph1015

Wang, W., Luo, R., Lin, Y., Wang, F., Zheng, P., Levi, M., et al. (2015). Aliskiren restores renal aqp2 expression during unilateral ureteral obstruction by inhibiting the inflammasome. Am. J. Physiol. Renal Physiol. 308 (8), F910F922. doi: 10.1152/ajprenal.00649.2014

Wu, W. P., Chang, C. H., Chiu, Y. T., Ku, C. L., Wen, M. C., Shu, K. H., et al. (2010). A reduction of unilateral ureteral obstruction-induced renal fibrosis by a therapy combining valsartan with aliskiren. Am. J. Physiol. Renal Physiol. 299 (5), F929-F941. doi: 10.1152/ajprenal.00192.2010
Yamaguchi, I., Tchao, B. N., Burger, M. L., Yamada, M., Hyodo, T., Giampietro, C., et al. (2012). Vascular endothelial cadherin modulates renal interstitial fibrosis. Nephron Exp. Nephrology 120 (1), e20-e31. doi: 10.1159/000332026

Yang, H. C., Zuo, Y., and Fogo, A. (2010). Models of chronic kidney disease. Drug Discovery Today Dis. Models 7 (1-2), 13-19. doi: 10.1016/j.ddmod.2010.08.002

Conflict of Interest: The authors declare that the research was conducted in the absence of any commercial or financial relationships that could be construed as a potential conflict of interest.

Copyright (C) 2019 Figueroa, Lozano, Lobos, Hennrikus, Gonzalez and Amador. This is an open-access article distributed under the terms of the Creative Commons Attribution License (CC BY). The use, distribution or reproduction in other forums is permitted, provided the original author(s) and the copyright owner(s) are credited and that the original publication in this journal is cited, in accordance with accepted academic practice. No use, distribution or reproduction is permitted which does not comply with these terms. 